\title{
Analysis, Synthesis, and Experimental Validation of a New Type of Microstrip Transition
}

\author{
F. Masot, F. Medina, and M. Horno, Member, IEEE
}

\begin{abstract}
This paper presents the theoretical analysis, synthesis, and experimental validation of a wideband transition between a conventional microstrip line and a microstrip line printed on a double-layer substrate. This transition is useful to link conventional microstrip circuits to certain special configurations fabricated on double-layer substrates. A particular case of generic interest is the transition between microstrip and suspended microstrip technologies. The analysis is carried out by using an enhanced version of the spectral domain analysis (SDA) that leads to computer codes quick enough to be used in an optimization design program.
\end{abstract}

\section{INTRODUCTION}

M ICROWAVE circuits using mixed single- and doublelayer microstrip technologies require the use of transitions between microstrips printed on two different supporting media. An example of this situation can be found in the transitions between conventional microstrip and suspended substrate microstrip technologies. Our interest in this transition arises from the study of the interconnection of a microstrip circuit on a single-layer substrate with a special directional coupler section recently proposed and studied in [1]. This coupler section makes use of a double-layer configuration.

In Fig. 1, we show the abrupt transition between two 50- $\Omega$ microstrip lines printed on two different media. These lines obviously have different widths. In spite of the fact that the characteristic impedance of the lines is the same at both sides of the transition, a big reflection coefficient is expected as a consequence of the strong mismatch between the transverse field patterns at each side. From a heuristic point of view, the performance of the discontinuity should be drastically improved by providing a smooth transition for the aforementioned field pattern. Smoothness could be achieved by means of a progressive change of the microstrip width from its value at the single-layer side, $w_{1}$, to its value on the double layer side, $w_{2}$. However, this gradual change has to be carried out in such a way that the local characteristic impedance, $Z_{c}(z)$, at any transverse $z$-plane of the transition region is still $50 \Omega$. Thus, the transition region should be made with a transmission line whose geometrical parameters could be changed in a continous way from the parameters of the line on the single-substrate region to the parameters of the line on the double-substrate region. A structure useful to achieve this purpose is the modified strip configuration whose

Manuscript received December 20, 1993; revised March 17, 1994. Thi work was supported by the DGICYT, Spain, Project No. TIC91-1018.

The authors are with the Departamento de Electronica y Electromagnetismo, Facultad de Física, Universidad de Sevilla, 41012 Sevilla, Spain.

IEEE Log Number 9406791.

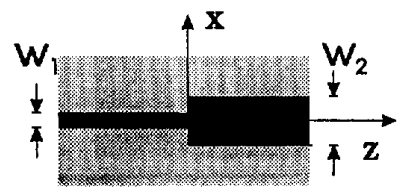

a)
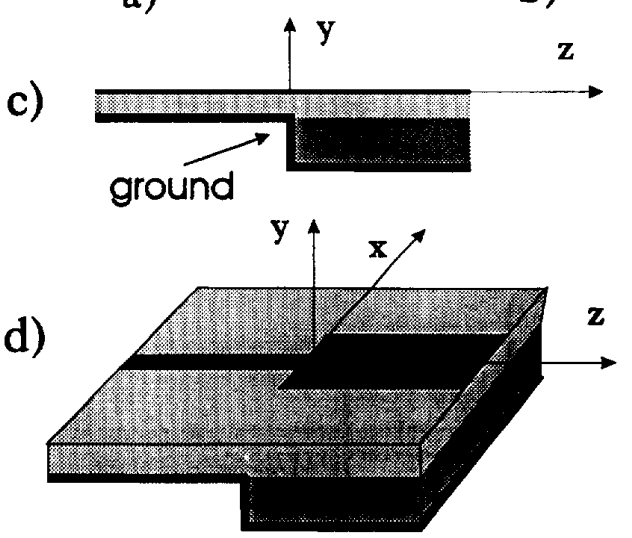

Fig. 1. Different perspectives of an abrupt transition between single- and double-layer microstrips. (a) Top view of the strip pattern on the upper side of the upper substrate. (b) Ground metallization of the bottom side of the upper substrate. (c) Side view of the abrupt transition. (d) Three-dimensional view.

cross-section is shown in Fig. 2. The strip and slot widths can be simultaneously modified as a function of $z(w(z)$ and $s(z)$ ) in such a way that the characteristic impedance of the strip-slot structure is $50 \Omega$ at any $z$-plane. The limits $s=0$ and $s=a$ correspond to the geometry of the lines actually present at each side of the transition (if the values of $w$ are properly chosen). This structure has been already used for the design of microstrip/slot directional couplers [2] (with $w$ and $s$ constant along the $z$-direction). Obviously, the $z$-profile for $w(z)$ is in some extent arbitrary-while keeping smoothness-if $s(z)$ is properly adjusted. One possible metallization pattern of the upper side of the substrate implementing this concept is shown in Fig. 3(a) (w varies linearly with $z$ in this case), and Fig. 3(b) shows the typical metallization shape of the bottom side. If the total length of the transition region is not too short, this structure provides a relatively low reflection transition (when compared with the abrupt transition in Fig. 1).

This paper also addresses the numerically efficient analysis and synthesis of the proposed transition. An accurate and quick computer code provides the characteristic propagation 


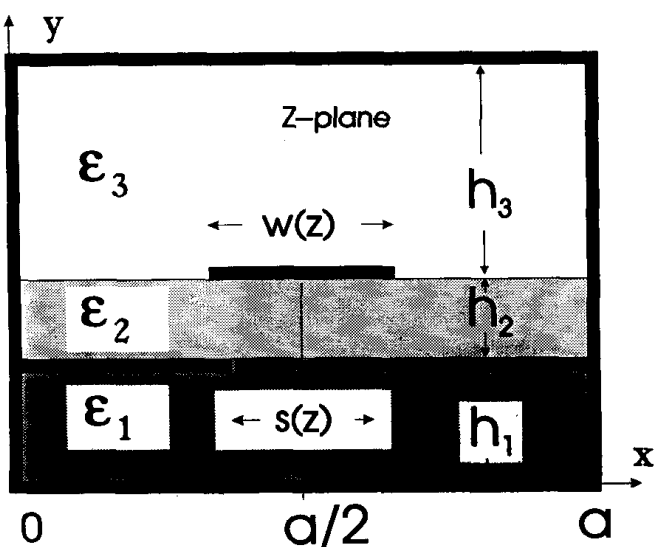

Fig. 2. Cross-section of the structure used for designing the smooth transition studied in this paper.

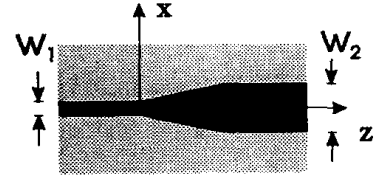

a)

c)

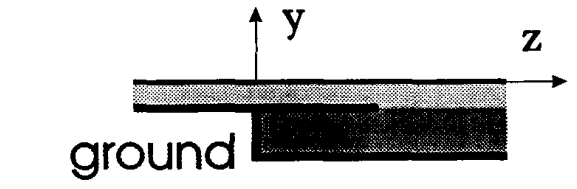

d)

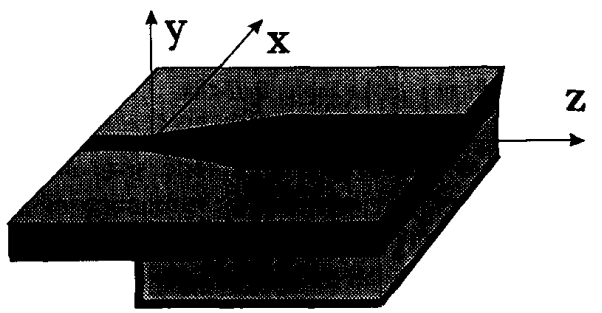

Fig. 3. Different perspectives of the smooth transition. (a) Top view of the upper metallized interface, (strip interface). (b) Top view of the lower interface (slot interface). (c) Longitudinal side view of the smooth interface. (d) Three-dimensional view.

parameters of the structure in Fig. 3. This program is used in a simple ad hoc optimization scheme which computes the value of $s$ for a given value of $w$ in such a way that the line has $50 \Omega$. Experiments have been carried out to show that the performance of the proposed transition is much better than of the abrupt transition.

\section{ANALYSIS/SYNTHESIS METHOD}

If we want to use the structure in Fig. 2 as a transition geometry, we have to solve for its characteristic impedance as a function of $w$ and $s$. This analysis can be easily carried out by means of the well-known SDA method. In order to achieve high numerical efficiency, we have implemented an enhanced version of the SDA under quasi-TEM assumptions. A structure having common features with the one analyzed in this paper was studied in [3]. The guidelines reported in that paper could be also followed to analyze the structure in Fig. 2. However, we have introduced some modifications in order to speed up the computations. Instead of writing an integral equation for the surface charge distribution on the strip $\left(\sigma_{2}(x)\right)$ and the slotted ground plane $\left(\sigma_{1}(x)\right)$, we write the integral equation for $\sigma_{2}(x)$ and the potential function at the slot $\left(v_{1}(x)\right)$. These quantities are linearly related to the surface charge distribution at the slotted ground plane $\left(\sigma_{1}(x)\right)$ and the potential function at the strip interface $\left(v_{2}(x)\right)$ as follows:

$$
\begin{aligned}
\sigma_{1}(x)= & \int_{\text {slot }} d x^{\prime} K_{11}\left(x, x^{\prime}\right) v_{1}\left(x^{\prime}\right) \\
& +\int_{\text {strip }} d x^{\prime} K_{12}\left(x, x^{\prime}\right) \sigma_{2}\left(x^{\prime}\right) \\
v_{2}(x)= & \int_{\text {slot }} d x^{\prime} K_{21}\left(x, x^{\prime}\right) v_{1}\left(x^{\prime}\right) \\
& +\int_{\text {strip }} d x^{\prime} K_{22}\left(x, x^{\prime}\right) \sigma_{2}\left(x^{\prime}\right)
\end{aligned}
$$

The sine Fourier transform of the previous integral equation yields its spectral domain version:

$$
\begin{aligned}
& \tilde{\sigma}_{1}\left(\alpha_{n}\right)=\tilde{K}_{11}\left(\alpha_{n}\right) \tilde{v}_{1}\left(\alpha_{n}\right)+\tilde{K}_{12}\left(\alpha_{n}\right) \tilde{\sigma}_{2}\left(\alpha_{n}\right) \\
& \tilde{v}_{2}\left(\alpha_{n}\right)=\tilde{K}_{21}\left(\alpha_{n}\right) \tilde{v}_{1}\left(\alpha_{n}\right)+\tilde{K}_{22}\left(\alpha_{n}\right) \tilde{\sigma}_{2}\left(\alpha_{n}\right)
\end{aligned}
$$

where ( $\sim$ ) stands for sine Fourier transform and $\alpha_{n}=n \pi / a$ is the Fourier variable.

The $\tilde{K}_{i j}\left(\alpha_{n}\right)$ elements in (2) are easily computed by following the spectral formulation in [4]. Thus, for the structure in Fig. 2 we can write

$$
\begin{aligned}
& \tilde{K}_{11}\left(\alpha_{n}\right)=\tilde{g}_{11}\left(\alpha_{n}\right)-\frac{\tilde{g}_{12}^{2}\left(\alpha_{n}\right)}{\tilde{g}_{22}\left(\alpha_{n}\right)} \\
& \tilde{K}_{12}\left(\alpha_{n}\right)=-\tilde{K}_{21}\left(\alpha_{n}\right)=\frac{\tilde{g}_{12}\left(\alpha_{n}\right)}{\tilde{g}_{22}\left(\alpha_{n}\right)} \\
& \tilde{K}_{22}\left(\alpha_{n}\right)=\tilde{g}_{22}^{-1}\left(\alpha_{n}\right)
\end{aligned}
$$

where the spectral functions $\tilde{g}_{i j}\left(\alpha_{n}\right)$ are [4]

$$
\begin{aligned}
\tilde{g}_{i i}\left(\alpha_{n}\right) & =\alpha_{n}\left[\varepsilon_{i} \operatorname{coth}\left(\alpha_{n} h_{i}\right)+\varepsilon_{i+1} \operatorname{coth}\left(\alpha_{n} h_{i+1}\right)\right] \\
\tilde{g}_{12}\left(\alpha_{n}\right) & =-\alpha_{n} \varepsilon_{2}\left[\sinh \left(\alpha_{n} h_{2}\right)\right]^{-1}
\end{aligned}
$$

Note that the possible anisotropy of the substrates can be accounted for as well as, if necessary, more complex multilayer configurations since the formulation in [4] is fully general.

As is well known, the per unit length (p.u.l.) capacitance and inductance of the transmission structure are obtained by solving (1) for the structure with and without dielectrics. The Galerkin method can be used to perform this task. Due to the physical nature of the expected solution, the following set of basis and test functions are used to approximate the slot potential, $v_{1}(x)$, and the strip surface charge density, $\sigma_{2}(x)$ :

$$
v_{1}(x)=\sum_{q=1}^{n_{1}} b_{q} f_{1, q}(x)=\sum_{q=1}^{n_{1}} b_{q} \frac{1}{\pi(2 q-1)} U_{2 q-1}\left(\frac{x-a / 2}{s / 2}\right)
$$


a)

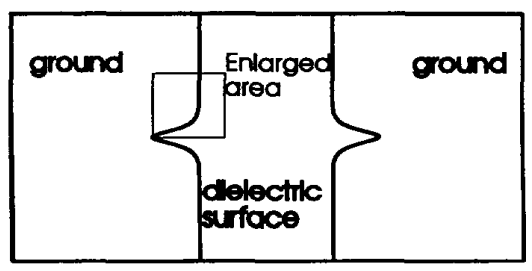

b)

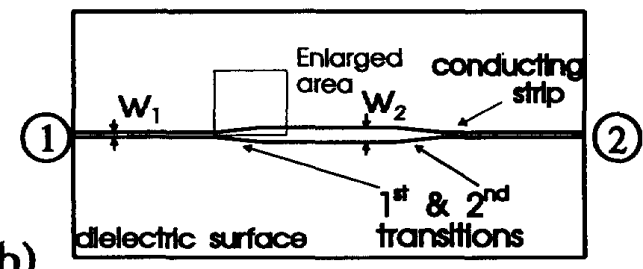

Fig. 4. Scaled layout of the measured smooth transition-(a) slot interface, (b) strip interface-connecting a single layer $\left(\varepsilon_{r}^{2}=10\right.$; thickness: $0.635 \mathrm{~mm}$ ) to a double-layer (subs. 1: $\varepsilon_{r}^{1}=2.42$; thickness: $0.49 \mathrm{~mm} \&$ subs. $2: \varepsilon_{r}^{2}=10$; thickness: $h_{1}=0.635 \mathrm{~mm}$ ) geometry. Strip widths: $w_{1}=0.63 \mathrm{~mm}$ (single layer); $w_{2}=1.86 \mathrm{~mm}$

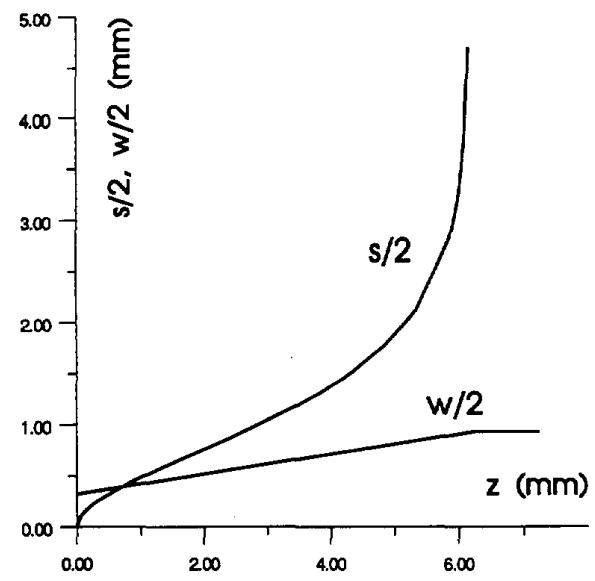

Fig. 5. Profile of the strip and slot metallization patterns of the smooth transition measured in this work.

$$
\begin{aligned}
\sigma_{2}(x) & =\sum_{p=1}^{n_{2}} a_{p} f_{2, p}(x) \\
& =\sum_{p=1}^{n_{2}} a_{p} \frac{2}{\pi w} \frac{T_{2(p-1)}\left(\frac{x-a / 2}{w / 2}\right)}{\left[1-\left(\frac{x-a / 2}{w / 2}\right)^{2}\right]^{1 / 2}}
\end{aligned}
$$

where $T_{n}(\cos \theta)=\cos (n \theta)$ and $U_{n}(\cos \theta)=\sin (n \theta)$ (i.e., first and second kind Chebyshev functions). This set of basis functions accounts for the symmetry of the structure and the metal edge condition for both quantities. As a consequence of this, extremely good convergence is achieved as the number of basis functions increases. Note that for this set of functions the p.u.l. capacitance is the coefficient $a_{1}$ in (5.2) when the strip voltage is the unity. The application of the Galerkin technique yields a linear equation system for $\left\{a_{p} ; p=1, \ldots, n_{2}\right\}$ and $\left\{b_{q} ; q=1, \ldots, n_{1}\right\}$ whose coefficients are defined either in terms of inner products/convolution integrals or simpler spectral series (thanks to the use of Parseval and convolution

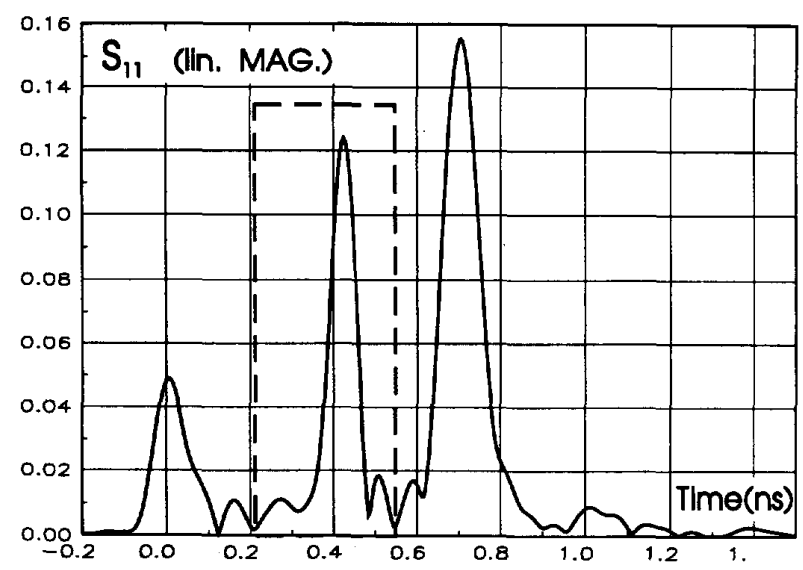

(a)

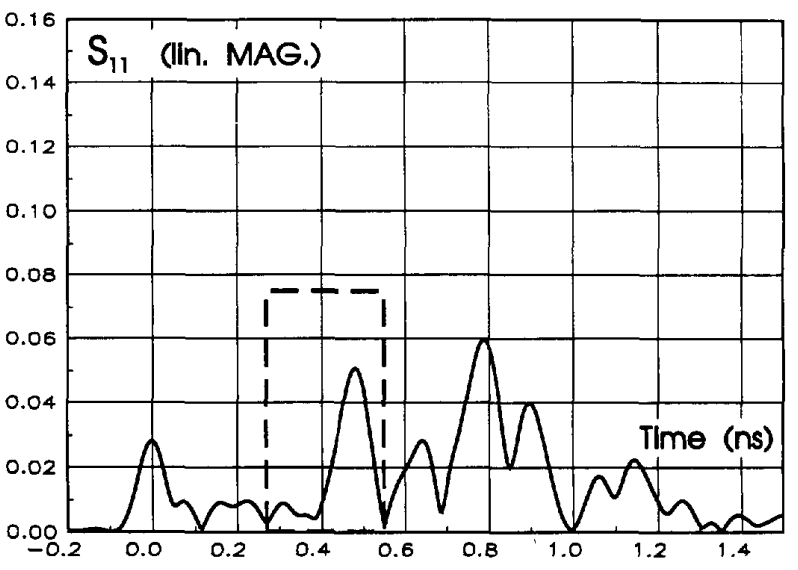

(b)

Fig. 6. (a) Time-domain return loss $\left(S_{11}\right)$ of the abrupt transition showing the gate used for de-embedding. (b) The same data for the smooth transition.

theorems). The latter representation is used because the general term of the Fourier series is known in closed form in terms of the Fourier transforms of the basis functions (5)--which are given in terms of Bessel functions-and the $\tilde{K}_{i j}\left(\alpha_{n}\right)$ functions in (2):

$$
A_{i, j}^{p, q}=\frac{2}{a} \sum_{n=1}^{\infty} \tilde{f}_{i, p}\left(\alpha_{n}\right) \cdot \tilde{K}_{i j}\left(\alpha_{n}\right) \cdot \tilde{f}_{j, q}\left(\alpha_{n}\right)
$$

The spectral series in (6) that involve $i \neq j$ show good exponential convergence, but the series involving interactions between basis functions defined on the same interface converge very slowly. Then, direct summation is very lengthy and is not suitable for quick and accurate design. Fortunately, the convergence can be drastically improved by means of the Kummer's method (extraction of the asymptotic tails). Note that for large values of the Fourier variable we have the following asymptotic limits:

$$
\begin{aligned}
& \tilde{K}_{11}^{\mathrm{as}}\left(\alpha_{n}\right)=\alpha_{n}\left(\varepsilon_{1}+\varepsilon_{2}\right) \\
& \tilde{K}_{22}^{\mathrm{as}}\left(\alpha_{n}\right)=\frac{1}{\alpha_{n}\left(\varepsilon_{2}+\varepsilon_{3}\right)}
\end{aligned}
$$


The asymptotic series obtained when we replace $\tilde{K}_{i i}\left(\alpha_{n}\right)$ by their asymptotic expressions (7) and use the Fourier transforms of the functions in (5) have the same form than the series considered in [5] in the context of the analysis of the single boxed microstrip line. Quasi-analytical expressions are reported there for those series (the reader is refered to that paper for more details). The remaining series (original series minus asymptotic series) converge exponentially in a few Fourier terms. The application of this technique makes the SDA extremely efficient from the computational point of view: the average CPU time for the analysis of typical lines is a fraction of one second on a $\mathrm{PC} / 386 / 25 \mathrm{MHz}$ platform with 80387 math coprocessor.

The previous analysis has been found useful for implementing a simple ad hoc optimization algorithm to design a gradual $50-\Omega$ microstrip-microstrip transition. The program automatically computes the slot width for a given strip width in order to keep the line impedance as close to $50 \Omega$ as specified. The error function $F Z(s)=Z_{s}-Z_{c}(w, s)\left(Z_{s}\right.$ is the standard impedance value, $50 \Omega$ in the usual case) is minimized for each value of $w(z)$. For the example reported in this paper, we have chosen a linear variation for the strip width, i.e. $w(z)=w_{1}+\left(w_{2}-w_{1}\right) \cdot(z / l)$, where $z$ is the coordinate of the propagation direction, $w_{1}$ and $w_{2}$ are the limits of $w$ on single $(w(z=0))$ and double layer $(w(z=l))$, and $l$ is the total length occupied by the transition area. Then, $s(z)$ is computed with the optimization program. The smooth behaviour of the error function, $F Z(s)$, permits one to employ a simple optimization method based on tangent outline, which typically converges within two or three iterations. The overall transition design process takes just severa' seconds on the aforementioned personal computer (in our designs $s(z)$ has been computed for twenty intermediate values of $w$, although five to ten points are enough from a practical point of view if they are properly distributed along the transition region).

\section{EXPERIMENTAL VALIDATION}

In addition to the theoretical analysis, we have carried out the experimental verification of our conclusions. Thus, we have fabricated a couple of transitions between a $50-\Omega$ microstrip line fabricated on Epsilam-10 and another 50- $\Omega$ microstrip line printed on a composite Epsilam-10/CuClad substrate. In fact, two transitions of each type have been cascaded in order to facilitate the measurement process. Figs. 4 and 5 show the dimensions and layout of the experimental setup used to measure the performance of the transition proposed in this work. A similar setup was built using abrupt transitions such as those shown in Fig. 1. Time domain postprocessing of the measured data permits one to calculate the parameters of the transitions under study. The measurements have been carried out using the HP-8510 ANA. The effect of coax-microstrip transitions has been partially removed from the measured results by using the method in [6], which exploits the time domain capabilities of the measurement system.

The reflection $\left(S_{11}\right)$ time domain results measured for the abrupt and the proposed (smooth) transitions are shown in Fig. 6(a) and (b), respectively. The portion of the time

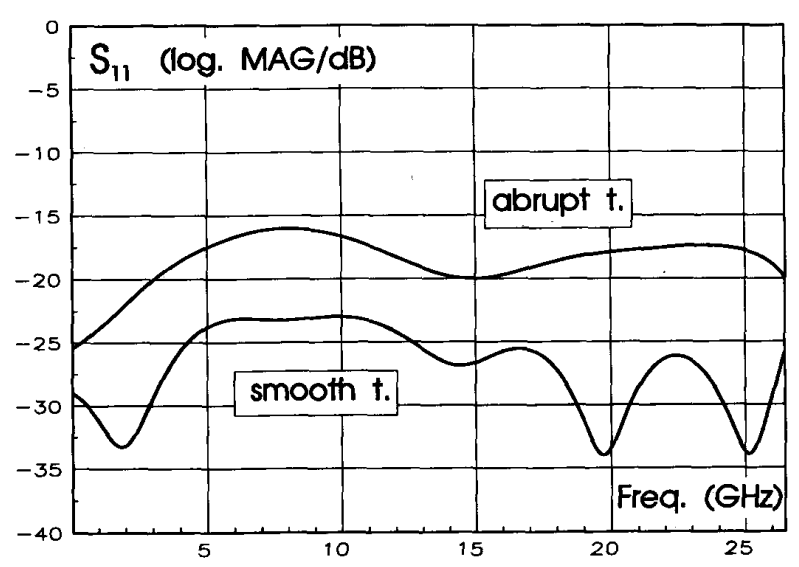

Fig. 7. Comparison of the $S_{11}$-response for the abrupt and smooth transitions.

response corresponding to the transition under study has been highlighted in those figures (the time gate has been included). After gating this part of the measured response and inverse Fourier transforming, we obtain the desired frequency domain performance of both transitions (see Fig. 7). The return loss of the proposed transition is good enough for most practical purposes in the whole frequency band considered in the measurement. However, the performance of the abrupt transition is rather poor.

\section{CONCLUSION}

In the present paper, we propose a transition geometry that could be useful when connecting double- and single-layer layouts in planar microstrip technology. The basic working principle of the proposed structure is that this structure provides smooth change of the transverse field pattern while keeping unchanged the nominal characteristic impedance. The control on the slot width allows us to get a transition as smooth as needed. The analysis of the transition section has been carried out using SDA techniques with a suitable math preproccesing. The analysis program gives very accurate results in fractions of one second ( $\mathrm{PC} / 386 / 25 \mathrm{MHz}$ platform). So, automatic design of the transition can be achieved in a few seconds on the abovementioned computer platform. Experimental verification of theoretical prediction has been included.

\section{REFERENCES}

[1] F. Masot, F. Medina, and M. Horno, "Analysis and experimental validation of a type of three strips microstrip coupler," vol. MTT-42, p. 1624-1635, Sept, 1994. IEEE Trans. Microwave Theory Tech

[2] M. Aikawa and H. Ogawa, "Double-sided MIC's and their applications," IEEE Trans. Microwave Theory Tech., vol. MTT-37, pp. 406-413, Feb. 1989.

[3] F. Masot, F. Medina, and M. Horno, "Accurate quasi-TEM analysis of modified suspended microstrip lines, and its application to directional coupler design," Microwave \& Opt. Tech. Lett.. vol. 4, no. 2, pp. 66-72, Jan. 1991.

[4] F. Medina and M. Horno, "Determination of Green's function matrix for multiconductor and anisotropic multidielectric planar transmission lines: a variational approach," IEEE Trans. Microwave Theory Tech., vol. MTT-33, pp. 933-940, Oct. 1985. 
[5] F. Medina and M. Horno, "Quasi-analytical static solution of the boxed microstrip line embedded in a layered medium," IEEE Trans. Microwave Theory Tech., vol. MTT-40, pp. 1748-1756, Sept. 1992.

[6] G. Gronau and I. Wolff, "A simple broad-band device de-embedding method using an automatic network analyzer with time-domain option," IEE Trans. Microwave Theory Tech., vol. MTT-37, pp. 479-483, Mar. 1989.

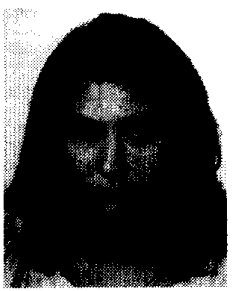

Fátima Masot was born in Badajoz, Spain, on January 29th, 1964. She received the Licenciada degree in physics from the University of Seville, Spain, in 1987. She had a scholarship of the Spanish Government and is currently assistant professor at the Applied Physics Department (University of Seville). Her research interests focus on the analysis of directional couplers and filters and other multiconductor line circuits.

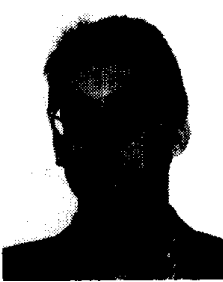

Francisco Medina was born in Puerto Real, Cádiz, Spain, on November, 1960 . He received the Licenciado degree in September 1983 and the Doctor degree in 1987, both in Physics, from the University of Seville, Spain. He spent the 1986-87 academic year at the Laboratoire de Microondes de l'ENSEEIHT (Toulouse, France) with a scholarship of MEC-MRT.

He was assistant professor at the Department of Electronics and Electromagnetics, University of Seville, during 1985-89. Since 1990, he has been associate professor of Electricity and Magnetism at the same institution. $\mathrm{He}$ has been a member of the Technical Programme Committee of the 23rd European Microwave Conference held in Madrid (Spain) on September, 1993. His research deals mainly with analytical and numerical methods for planar structures and applications of multiconductor lines.

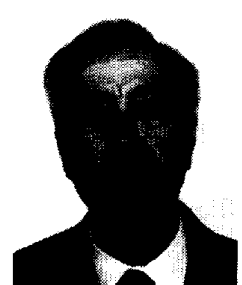

Manuel Horno (M'75) was born in Torre del Campo, Jaén, Spain. He received the Licenciado degree in Physics in June 1969, and the Doctor degree in Physics in January 1972, both from the University of Seville, Spain.

Since October 1969 he has been with the Department of Electronics and Electromagnetism at the University of Seville, where he became an assistant professor in 1970, associate professor in 1975 , and professor in 1986. He is a member of Electromagnetism Academy of MIT (Cambridge. MA). His main fields of interest include boundary value problems in electromagnetic theory, wave propagation through anisotropic media, and microwave integrated circuits. He is presently engaged in the analysis of planar transmission lines embedded in complex materials, multiconductor transmission lines, and printed antennas. 